Ein praktikables Instrument zum Vergleich von Unternehmenskonzepten

\section{Das ASU-Benchmarking-System}

\section{Die Arbeitsgemeinschaft selbstständiger Unternehmer (ASU) hat 1988 erst- mals einen Preis für umweltorientierte Unternehmensführung entwickelt. Die Bewerber werden anhand eines Benchmarking-Systems bewertet. Die Unter- nehmen können dem System Hinweise für die Weiterentwicklung ihrer umweltorientierten Unternehmenskonzepte entnehmen.}

I

Von Klaus Günther

mmer mehr Firmen entwickeln umweltori-

entierte Unternehmenskonzepte, die sowohl ökologische wie auch ökonomische Ziele verbinden. Sie haben realisiert, daß die Umwelt gewissermaßen als moderne und ganzheitliche Version des Produktionsfaktors „Boden“ einen Engpaß darstellt, der gegenüber „Kapital“ und „Arbeit“ zunehmend an Bedeutung gewinnt und im Managementsystem angemessen zu berücksichtigen ist. Unterschiedliche Ansätze und Instrumente werden erprobt und dokumentiert, so daß es inzwischen durchaus an der Zeit ist, sich mit den Stärken- und Schwächenprofilen solcher Unternehmenskonzepte auseinanderzusetzen und diese mit denen anderer Unternehmen zu vergleichen, um schließlich eine Meßlatte zur Orientierung zu definieren.

\section{- Entwicklung des ASU-Öko-Benchmarking}

Die Stärken- und Schwächenprofile umweltaktiver Unternehmen, über die hier berichtet wird, wurden bei den Bewerbern um den „ASU-Preis für umweltorientierte Unternehmensführung" ermittelt. 1987 konzipiert und 1988 erstmals vergeben, ist er einer der ersten Umweltpreise dieser Art. Seit 1988 wurde der ASU Umweltpreis nach laufend verschärften Kriterien viermal vergeben. Bei der Ausschreibung für die Umweltpreise 1994 war die im April 95 wirksam gewordene „EG-Öko-Audit-Verordnung“ Anlaß, eine Prüfliste zu entwickeln, die die Anforderungen dieser Verordnung in einem Fragenkatalog abbildete. Dabei lag das Augenmerk der ASU weniger in einer Beteiligung am Zertifizierungsverfahren, sondern in allererster Linie in der Förderung von praktikablen Umweltmanagementkonzepten (,zertifizierungsfähig“ werden ist das Hauptziel) im Sinne von ,sustainable development".

Die Bewerber wurden aufgefordert, auf 50 Fragen zu den Unternehmensthemen Umweltpolitik als Teil der Unternehmenspolitik, Umweltziele, Umweltprogramm und Umweltmanagementsystem $\mathrm{zu}$ antworten. Bei den Fragen zum Umweltmanagementsystem wurden Angaben zu Organisation, Kommunikation, Umweltschutz in der Aus- und Weiterbildung, Steuerung und Kontrolle, Risikomanagement, Beschaffung, Logistik, Produktion und Betriebsabläufe, Produktmanagement und natürlich über das Abfallmanagement erwartet.

Man erkennt in diesen Punkten unschwer die Kriterien in Anhang I der EG-Öko-Audit-Verordnung. Sie wurden hier etwas griffiger und praxisnäher formuliert.

In einer Erklärung mußten die Bewerber für den ASU-Preis weiter bestätigen:

- daß kein Umweltstrafverfahren anhängig ist,

- daß das Unternehmen sich verpflichtet, die Urkunde betriebsüblich bekanntzugeben (internes Controlling durch Mitarbeiter),

- daß das Unternehmen im Rahmen der ASUÖffentlichkeitsarbeit sein Ökokonzept publiziert (öffentliche Kontrolle),

- und auch Dritten konkret Auskunft zu Einzelfragen gibt (Multiplikatoreffekt)

Unter dem Strich also eine Vorform der Umwelterklärung. Die Resonanz auf die umfangreiche

\section{Die zentralen Kennzeichen des} ASU-Umweltpreises

Insgesamt zeichnet sich der ASU-Preis durch Kontinuität, hohes Niveau und Seriösität aus:

- Die ASU vergibt lediglich eine Urkunde und keine Geld preise.

- Der Wettbewerb wendet sich an alle Unternehmen, nicht nur an Mitglieder.

Im Mittelpunkt stand von Anfang an nicht die Honorierung einer ökologisch hervorragenden Einzelleistung, 2.B. eine Produktentwicklung, sondern der Nachweis eines praktizierten, ganzheitlich umwelt-orientierten Unternehmenskonzeptes.

- Die Bewertung wurde von einem neutralen Gremium unter wissenschafllicher Leitung durchgeführt
Prüfliste erfïllte die Erwartungen. Insbesondere konnte gegenüber den vorangegangenen Aktionen ein weiterer Qualitätsanstieg der eingereichten Unterlagen festgestellt werden. Nicht wenige Unternehmen legten kiloschwere Aktenordner vor. Eine große Zahl von Unternehmen hat die Prüfliste zum internen Check genutzt.

\section{- Auswertungsverfahren}

Die Berücksichtigung des Aspekts der Veränderung war ein sehr wichtiger Punkt für die Gewichtung der einzelnen Bewertungsfaktoren Dabei wurde der Erfahrung Rechnung getragen daß menschliches Verhalten nur dann nachhaltig verändert werden kann, wenn es gelingt, das Denken der einzelnen zu verändern. Integriertes Umweltmanagement verlangt sowohl die Berïcksichtigung der sachbezogenen Bereiche Technik und Betriebswirtschaft als auch personenbezogene/emotionale Aspekte, wie Unternehmenskultur, Kommunikation, Mitarbeiterschulung und Motivation.

Dieser Dualismus, der gleich wichtigen und permanenten Beruicksichtigung ,harter" und ,weicher" Faktoren innerhalb des gesamten Beziehungsgeflechtes eines Unternehmens, ist ein entscheidender Erfolgsfaktor. Entsprechend wurden die Aspekte beim Benchmarking in etwa gleich gewichtet.

Die Auswertung erfolgte unter wissenschaftlicher Leitung (Uni Oldenburg, IÖW). Die eingereichten Unterlagen wurden nach einem Punkteverfahren bewertet. Ergebnis war eine Rankingtabelle, in der alle Bewerber mit ihrer Punktzahl aufgeführt wurden. Nach Abschluß der Einzelbewertung wurde eine Darstellungsform gesucht, die es dem Unternehmer ermöglicht, sein Umweltmanagementsystem besser einzuordnen. Für ihn ist die Bewertung für den ASU-Preis in der Regel kein Selbstzweck. Ihn bewegen vielmehr praktische Fragen, wie: Wo steht mein Unternehmen in bezug auf die EGÖko-Audit-VO oder im Verhältnis zu anderen Unternehmen? Welche Defizite bestehen? Wo besteht Handlungsbedarf?

\section{- Enwicklung des Benchmarking}

Und hier schlug dann die Stunde des Benchmarking, denn dieses Verfahren bietet sich für eine erste Einschätzung an. Alle Bewerber erhielten daher eine grafische Auswertung ihrer Bewerbungsunterlagen. In diesem ASU-ÖkoBenchmarking können sie ablesen, wie sie im Vergleich zu dem Anforderungen der Prüfliste 
(angelehnt an EG-Öko-Audit-Verordnung, deren Erfïllung gewissermaßen als „Bestmarke“ definiert wird) und im Vergleich zum Durchschnitt aller Bewerber abgeschnitten haben.

Nach Auswertung und Aggregation aller eingereichten Bewerbungsunterlagen steht der ASU nun eine Datenbasis zur Verfügung, die in Deutschland wohl bisher einmalig ist. Die Analyse der bisher vorliegenden Einzeldaten brachte folgende erste Ergebnisse zutage. Da die Bewerber durchweg umweltaktive Unternehmen sind, erstaunt es kaum, daß die Durchschnittskurve relativ hoch angesiedelt ist. Als zum Teil noch unterentwickelt erscheinen folgende Bereiche:

- Umweltziele: Lücken gibt es, so zeigt die Auswertung, bei der zeitlichen und quantitativen Formulierung von Zielvorgaben. Diese aber sind erforderlich, um periodisch einen aussagefähigen Soll/Ist-Vergleich zu machen.

- Umweltprogramm: Oftmals findet bei den Bewerbern keine vernünftige Maßnahmenplanung zur Verwirklichung der Umweltziele statt. Vielmehr herrscht hier noch das ,Zufalls“-Prinzip. Möglicherweise sind aber auch die Begriffe und Definitionen der Verordnung für Mittelständler zu theoretisch und kaum in der reinen Form praktikabel. So ist die formale Trennung von Umweltprogramm und Umweltzielen für viele kaum einsehbar.

- Kommunikation: Viele Unternehmen scheuen den Kontakt mit der Öffentlichkeit. So bleibt ein wichtiges PR-Instrument ungenutzt, das nicht zuletzt geeignet ist, die sogenannten ,stakeholder relations" zu verbessern. Dabei brauchen die meisten der beteiligten Unternehmen sich mit ihrem Umweltmanagement nicht $\mathrm{zu}$ verstecken. Nach dem Motto „Tue Gutes und rede darüber" sollten die Unternehmer offensiv an die Öffentlichkeit gehen.

- Steuerung und Kontrolle: Unsere Auswertungen haben ergeben, daß bei vielen Unternehmen noch Defizite bei der Steuerung und Kontrolle über die Umsetzung der Umweltpolitik vorhanden sind. Vielfach fehlt noch ein systematisches Ökocontrolling, da die Instrumente für die Erfassung der Stoff- und Energieströme nur partiell vorhanden sind. Auch bei der ökologischen Bewertung von bezogenen Vorleistungen und Rohstoffen wurden Lücken festgestellt.

- Produktmanagement: Während das Management fast überall den Einsatz von umweltgerechten Verpackungen fördert, sind ökologische Betrachtungen des gesamten Produktlebenszyklus bei vielen Unternehmen noch nicht verbrei-

\section{Bei der ASU-Befragung in den Bereichen erzielte Punkte nach Unternehmensgrößen}

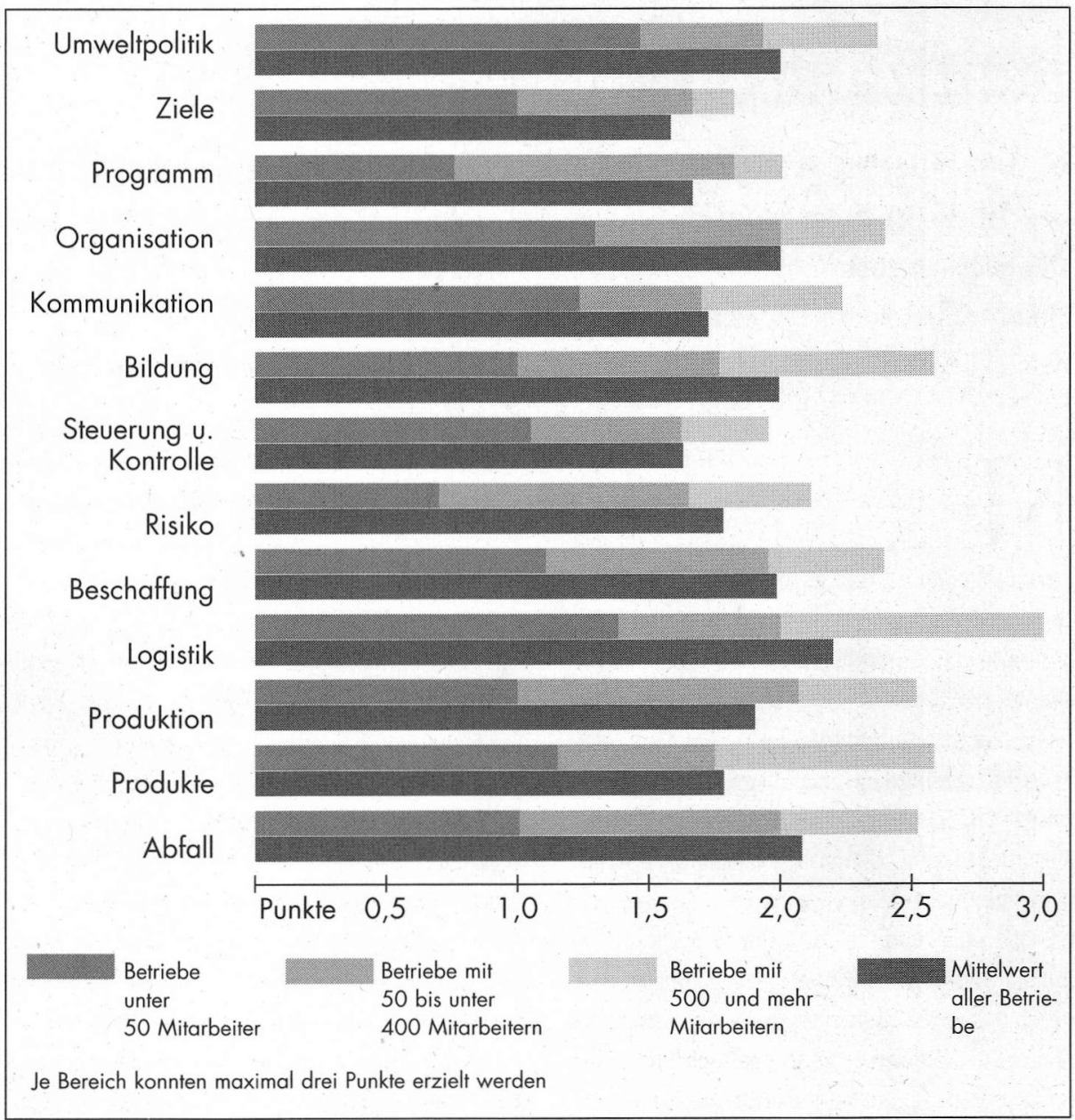

tet. Auch machen sich zu wenige Unternehmen Gedanken, was mit ihren Produkten geschieht, wenn sie ausgedient haben und auf dem Abfallberg landen oder in der Nordsee versenkt werden sollen. Hier sind die ökologischen Hausaufgaben bisher noch nicht genügend erledigt.

\section{- Erste Schlußfolgerungen}

Welche Schlußfolgerungen sind nun aus diesen Ergebnissen zu ziehen? Sind die Großunternehmen wirklich ökologisch erfolgreicher? Oder beherrschen sie den (notwendigen?) Formalismus besser? Besteht im Mittelstand ein erhöhter Beratungsbedarf? Detailliertere Analysen werden hier wertvolle Anstösse für die weitere Arbeit auch für Verbände, Wissenschaft, Politik usw. geben. Sicher scheint mir zu sein, daß die Qualität und Effektivität von betrieblichen Umweltkonzepten in diesem Kontext eine zunehmend wichtige Rolle spielen wird. Notwendig sind meßbare Ziele und konkrete Maßstäbe, etwas was in den USA als ,ecological performance evaluation“ bezeichnet wird. Wir werden in der ASU daher nicht nur diesen ersten, zuge- gebenermaßen noch holprigen Versuch eines Öko-Benchmarking weiterentwickeln (die Ausschreibung des ASU-Preises 1996 wird z.Zt. unter weiter qualifizierten Bedingungen vorbereitet), sondern auch ganz konkret Unterstiitzung bei der betrieblichen Entwicklung und Einführung von Umweltmanagementsystemen geben.

Ökobenchmarking scheint uns ein probates, praktikables und transparentes Instrument $\mathrm{zu}$ sein, um den Status von Umweltmanagement glaubwürdig zu dokumentieren und Hinweise für die notwendigen und richtigen nächsten Schritte zur Weiterentwicklung umweltorientierter Unternehmenskonzepte im Sinne von sustainable developement zu erhalten.

\section{Der Autor}

Klous Günther ist geschäftsführender Gesellschafter der Günther GmbH + $\mathrm{Co}$ in Lengerich/Westfalen (Kunsisioff- und Glasverarbeitung), Vizepräsident der ASU und Vorstand des future e.V.

Kontakt: Günther GmbH \& Co, Posifach 1270 49512 Lengerich 
(c) 20I0 Authors; licensee IÖW and oekom verlag. This is an article distributed under the terms of the Creative Commons Attribution Non-Commercial No Derivates License (http://creativecommons.org/licenses/by-nc-nd/3.o/), which permits unrestricted use, distribution, and reproduction in any medium, provided the original work is properly cited. 